\title{
DIGESTIBILIDADE DE DIETAS COM DIFERENTES FONTES DE CARBOIDRATOS E SUA INFLUÊNCIA NA GLICEMIA E INSULINEMIA DE CÃES
}

\author{
Digestibility of diets with different sources of carbohydrates and your influence in the blood glucose \\ and blood insulin in health dogs
}

\author{
José Walter da Silva Júnior ${ }^{1}$, Flávia Maria de Oliveira Borges ${ }^{2}$, Luis David Solis Murgas ${ }^{3}$, \\ Ana Gabriela Valério ${ }^{4}$, Guilerme Coelho Medeiros ${ }^{4}$, Renata Viana ${ }^{4}$, Lídia Marinho Silva Lima ${ }^{4}$
}

\begin{abstract}
RESUMO
Com o objetivo de estudar a influência das dietas com diferentes fontes de carboidratos na glicemia e insulinemia pós-prandial de cães, foi conduzido, no Departamento de Zootecnia da Universidade Federal de Lavras (UFLA), um experimento com cães da raça Foxhound Americano. Foram utilizados 24 cães machos com peso médio de 34,61 $\pm 3,42 \mathrm{~kg}$, distribuídos em delineamento em blocos casualizados, com parcela subdividida no tempo, com quatro tratamentos, e um animal por unidade experimental. Os tratamentos consistiram em três dietas, formuladas para conterem as mesmas quantidades (63\%) dos cereais avaliados (milho, sorgo e arroz) e uma quarta dieta constituindo uma mistura em partes iguais de cada cereal. As dietas foram isoenergéticas e isoprotéicas. Os animais foram alimentados uma vez ao dia, pela manhã, e as quantidades ingeridas foram estabelecidas de acordo com a equação de predição de Heusner (1982) para energia de manutenção acrescidas em 10\%. Foram avaliadas a digestibilidade da matéria seca, a digestibilidade do amido total, a energia digestível, a glicemia e a insulinemia. Para a diferenciação das médias, foi utilizado o teste de Scott-Knott $(\mathrm{P}<0,05)$. Observou-se que a dieta 3 (arroz) foi superior $(\mathrm{P}<0,05)$ para a variável digestibilidade da matéria seca e digestibilidade do amido total. Para a variável energia digestível, as dietas 1 e 3 (milho e arroz) foram superiores às demais $(\mathrm{P}<0,05)$. Não foi observada diferença significativa entre as dietas para as variáveis glicemia $(\mathrm{P}=0,51)$ e insulinemia $(\mathrm{P}=0,66)$ pelo teste $\mathrm{F}$. Concluiu-se que as diferentes fontes de carboidratos utilizadas nas quatro dietas não influenciaram a glicemia e insulinemia pós-prandial em cães sadios.
\end{abstract}

Termos para indexação: glicemia, insulinemia, digestibilidade, cães, amido, carboidratos.

\begin{abstract}
In order to evaluate the influence of the diets with different carbohydrates sources in blood glucose and blood insulin in dogs, were conducted, in Animal Science Department of Federal University of Lavras (UFLA), a experiment with dogs of breed American Foxhound. Were utilized 24 males dogs with average weight of the 34,61 $\pm 3,42 \mathrm{~kg}$, allotted in randomized blocks design with subdivided piece in time, with four treatments, and one animal per unit experimental. The treatments were three diets formulated to contain the same amount (63\%) of the cereals evaluate (corn, sorghum and rice) and a forth diet being a blend in equal parts of each cereal. The animals were fed once per day, for the morning, and the consume established agreed with the prediction equation of the Heusner (1982) for mantence energy, plus 10\%. The digestible dry matter, the digestible starch, the digestible energy, the blood glucose and blood insulin were evaluated. For the statistical analysis were utilized the test of Scort-Knott $(\mathrm{P}<0,05)$. Thus, the diet 3 (rice) were higher $(\mathrm{P}<0,05)$ for the digestibility of dry matter variable and digestible starch variable. For the digestible energy variable, the diets 1 and 3 (corn and rice) were higher then others $(\mathrm{P}<0,05)$. It was found that the treatments didn't influence the blood glucose $(\mathrm{P}=0,51)$ and blood insulin $(\mathrm{P}=0,66)$ for test $\mathrm{F}$. In conclusion, the different sources of the carbohydrates utilized, in the four diets, didn't influence in the blood glucose and blood insulin in health dogs.
\end{abstract}

Index terms: blood glucose, blood insulin, dog, starch, carbohydrates.

(Recebido para publicação em 27 de abril de 2004 e aprovado em 12 de Janeiro de 2005)

\section{INTRODUÇÃO}

A nutrição adequada está diretamente relacionada à melhor qualidade de vida e é determinante da longevidade do animal. Um cão alimentado com uma dieta corretamente balanceada possui melhores chances de vencer os desafios impostos à sua saúde, desfrutando de uma vida mais saudável por mais tempo.
Hoje, se discute que uma das causas possíveis de doenças, como insuficiência renal e insuficiência cardíaca, seria a hipertensão arterial causada por uma inabilidade de excreção do sódio, que ficaria retido no organismo devido ao excesso de insulina. Esse hiperinsulinismo é provocado pelo consumo contínuo de dietas ricas em carboidratos e açúcares (BEHREND \& GRECO, 2000a; TER MAATEN et al., 1997).

1. Médico Veterinário - Especialista em Residência Clinica de cães e gatos - Mestre e Doutorando em nutrição de cães e gatos - zewalter77@yahoo.com.br

2. Professora Adjunta do Departamento de Zootecnia da Universidade Federal de Lavras/UFLA - Caixa Postal 3037 - 37.200-000 - Lavras, MG - borgesvet@ufla.br

3. Professor Adjunto do Departamento de Medicina Veterinária/UFLA.

4. Alunos do $10^{\circ}$ período de Medicina Veterinária/UFLA. 
Além de reter sódio, a relação insulina/glucagon é responsável pela modulação das enzimas do metabolismo de carboidratos e lipídeos, de modo que uma alta relação insulina/glucagon, geralmente no período pós absortivo, exacerba as vias de síntese de glicogênio e lipídeos, ao passo que uma baixa relação insulina/glucagon levaria o organismo a ativar vias de liberação de energia como lipólise e gliconeogênese. Dessa forma, o animal que conseguir manter a relação insulina/glucagon predominantemente baixa dificilmente terá problemas, como obesidade, diabetes, doenças cardíacas e respiratórias (BEHREND \& GRECO, 2000a; TER MAATEN et al., 1997).

Há vários métodos de mensuração da glicemia. O método referencial é realizado em laboratório, geralmente utilizando a enzima glicose oxidase (PEREIRA et al., 2003). Outros métodos utilizados são fitas reagentes com variação de cor, "color test strips", ou monitores de glicose portáteis (COHN et al., 2000). As vantagens no uso de monitores portáteis de glicose são muitas. A praticidade, os baixos custos e a velocidade na obtenção de resultados são algumas delas. Entretanto, a mais evidente vantagem é a necessidade de apenas uma pequena quantidade de sangue, que pode ser obtida sem que o animal fique estressado. Assim, evita-se que ocorra uma hiperglicemia momentânea devido ao estresse, como tem sido observado em felinos (BEHREND \& GRECO, 2000b; RAND, 1997; TORR et al., 1991).

Realizou-se este experimento com o objetivo de avaliar a digestibilidade de quatro tipos diferentes de dietas à base de cereais e sua resposta glicêmica e insulinêmica em cães, buscando identificar qual cereal de importância industrial (milho, sorgo, arroz ou mistura desses) possui menor influência sobre a glicemia e insulinemia dos cães.

\section{MATERIAL E MÉTODOS}

O experimento foi realizado na cidade de Três Corações, sul de Minas Gerais, Região Sudeste do Brasil. Foram utilizados cães da raça Foxhound Americano, machos, não castrados, com idade média de 03 anos, alojados em canil individual nas instalações da estação experimental da empresa Total Alimentos S/A. Foram utilizados 24 cães, divididos em 4 tratamentos com 6 animais cada um. O peso médio dos animais foi de $34,61 \pm 3,42 \mathrm{~kg}$.

Os cereais avaliados foram: o milho, o sorgo, a quirera de arroz e uma mistura em partes iguais dos três cereais. Cada cereal avaliado foi incluído em uma porcentagem de $63 \%$, na base da matéria natural, à dieta, a qual constitui um tratamento avaliado.

As dietas experimentais foram formuladas para que não houvesse diferença entre as porcentagens de proteína bruta (PB), extrato etéreo (EE), fibra bruta (FB), matéria mineral (MM) e extrato não nitrogenado (ENN), sendo consideradas, portanto, isoenergéticas e isoprotéicas. O objetivo foi avaliar os cereais; por isso, eles entraram em proporções idênticas nas três dietas. A quarta dieta foi formulada para verificar as possíveis interações e efeitos aditivos da mistura dos cereais. Na Tabela 2 verifica-sea a formulação das dietas e na Tabela 1, a composição química calculada das dietas a partir das formulações.

TABELA 1 - Composição química das dietas, em porcentagem da matéria seca, calculada a partir da formulação.

\begin{tabular}{lcccccccc}
\hline \multicolumn{1}{c}{ Dietas } & MS & $\begin{array}{c}\text { Amido } \\
\text { Total } \\
(\mathbf{A T})\end{array}$ & ENN & PB & EE & FB & MM & $\begin{array}{c}\text { Energia Metabolizável } \\
\text { (EM) calculada }\end{array}$ \\
& $(\%)$ & $(\%)$ & $(\%)$ & $(\%)$ & $(\%)$ & $(\%)$ & $(\%)$ & $\mathrm{Kcal} / \mathrm{kg}$ \\
\hline Dieta 1 (milho) & 100,00 & 48,38 & 59,10 & 22,67 & 9,74 & 1,73 & 6,77 & 3689 \\
Dieta 2 (sorgo) & 100,00 & 44,88 & 60,24 & 22,45 & 8,76 & 1,93 & 6,62 & 3639 \\
Dieta 3 (arroz) & 100,00 & 41,63 & 62,47 & 22,64 & 7,67 & 0,67 & 6,56 & 3630 \\
Dieta 4 (mistura) & 100,00 & 45,67 & 60,60 & 22,59 & 8,72 & 1,45 & 6,64 & 3653 \\
\hline
\end{tabular}


Os animais foram alimentados uma vez ao dia. A quantidade de ração fornecida foi controlada e calculada de acordo com as necessidades energéticas de cada animal, determinadas por meio da equação de predição de Heusner (1982), citado no NRC (1985), acrescida de 10\%. O período total de adaptação à dieta foi de 15 dias. Além das dietas experimentais, os cães receberam água fresca à vontade.

Foi realizada colheita total de fezes por um período de cinco dias consecutivos. As colheitas de sangue foram realizadas por meio de punção da veia cefálica dos cães, com o uso de seringa de $10 \mathrm{~mL}$ e agulha de $30 \times 0,8 \mathrm{~mm}$., segundo Nogueira et al. (2002). Para cada cão, foram colhidas 17 amostras de sangue para a mensuração da glicemia, sendo a primeira em jejum e as demais nos tempos: 0,25 hora; 0,5 hora; 0,75 hora; 1,0 hora; 1,5 hora; 2 horas; 2,5 horas; 3 horas; 3,5 horas; 4,5 horas; 5,5 horas; 6,5 horas; 7,5 horas; 8,5 horas; 9,5 horas e 10,5 horas após a alimentação. Para a mensuração da insulinemia, foram colhidas 08 amostras, sendo a primeira em jejum e as demais nos tempos 0,25 hora; 0,5 hora; 0,75 hora; 1,0 hora; 1,5 hora; 2 horas e 3 horas, após a alimentação. Colheram-se ainda amostras de cada dieta análises químicas.

As análises foram realizadas no Laboratório de Pesquisa Animal do Departamento de Zootecnia da UFLA, MG, com exceção da determinação do amido total pelo método de Nelson (1944) e a determinação do teor de extrato etéreo das amostras, realizada por hidrólise ácida (AOAC, 1984), as quais foram realizadas no Laboratório de Produtos Vegetais do Departamento de Ciência dos Alimentos da UFLA, MG.

A glicemia foi determinada utilizando-se um glicosômetro portátil, ou monitor portátil de glicose, o "Accu-Chek ${ }^{\circledR}$ Advantage". A insulinemia foi determinada por doseamento imunométrico com o analisador IMMULITE $^{\circledR} 2000$.

TABELA 2 - Formulação das dietas experimentais, valores em porcentagem na base da matéria natural.

\begin{tabular}{lcccc}
\hline \multicolumn{1}{c}{ Ingredientes } & Dieta 1 & Dieta 2 & Dieta 3 & Dieta 4 \\
\hline Milho grão & 63,0 & -- & -- & 21,0 \\
Sorgo grão & -- & 63,0 & -- & 21,0 \\
Quirera de arroz & -- & -- & 63,0 & 21,0 \\
Farinha de vísceras integral & 15,0 & 15,0 & 15,0 & 15,0 \\
Farelo de soja & 5,0 & 5,0 & 5,0 & 5,0 \\
Farinha de carne (45\%) & 8,0 & 8,0 & 8,0 & 8,0 \\
Sal branco comum & 0,5 & 0,5 & 0,5 & 0,5 \\
Açúcar & 0,5 & 0,5 & 0,5 & 0,5 \\
Palatabilizante & 4,0 & 4,0 & 4,0 & 4,0 \\
Gordura animal & 3,0 & 3,0 & 3,0 & 3,0 \\
Complexo Mineral/Vitamínico & 1,0 & 1,0 & 1,0 & 1,0 \\
\hline Total & 100,0 & 100,0 & 100,0 & 100,0 \\
& & & & \\
\hline
\end{tabular}

Enriquecimento por quilograma de produto

14.000 U.I de vitamina a, $8 \mathrm{mg}$ de vitamina b1, $200 \mathrm{mcg}$ de vitamina b12, $8 \mathrm{mg}$ de vitamina b2, 8 mg de vitamina b6, 2.000 u.i. de vitamina d, 80 u.i. de vitamina e, $1,5 \mathrm{mg}$ de vitamina h, 1,5 mg de vitamina $\mathrm{k}, 60 \mathrm{mg}$ de vitamina pp, $120 \mathrm{mg}$ de zinco, 1,5 mg de ácido fólico, $20 \mathrm{mg}$ de ácido pantotênico, $1150 \mathrm{mg}$ de colina, $15 \mathrm{mg}$ de cobre, $0,5 \mathrm{mg}$ de cobalto, $125 \mathrm{mg}$ de etoxiquin, $170 \mathrm{mg}$ de ferro, $3 \mathrm{mg}$ de iodo, $1 \mathrm{~g}$ de magnésio, $80 \mathrm{mg}$ de manganês, $5000 \mathrm{mg}$ de potássio, $0,2 \mathrm{mg}$ de selênio, $3,5 \mathrm{~g}$ de sódio. 
As variáveis foram analisadas segundo o pacote "SISVAR for Windows" versão 4.6 (FURTADO, 2003). $\mathrm{Na}$ verificação de diferença significativa entre as variáveis, o teste de Scott-Knott $(\mathrm{P}<0,05)$ foi utilizado para classificação de médias. Cada parcela experimental foi constituída de um cão. O delineamento experimental utilizado para as variáveis digestibilidade da matéria seca, digestibilidade do amido total e energia digestível das dietas foi o de blocos inteiramente casualizados, com quatro tratamentos e seis repetições. Para as variáveis glicemia e insulinemia, o delineamento experimental utilizado foi o de blocos inteiramente casualizados, com parcelas subdivididas no tempo, sendo o modelo estatístico:

$$
Y_{i j}=\mu+a_{i}+r_{j}+e_{i j} \text {, em que: }
$$

$\mathrm{Y}_{\mathrm{ij}}=$ observação da variável referente a parcela $\mathrm{i}$ no bloco j;

$\mu=$ média geral;

$\mathrm{a}_{\mathrm{i}}=\mathrm{o}$ efeito da dieta $\mathrm{i}(\mathrm{i}=1,2,3,4)$;

$r_{j}=$ o efeito do bloco $\mathrm{j}(\mathrm{j}=1,2,3,4,5,6)$;

$\mathrm{e}_{\mathrm{ij}}=\mathrm{o}$ erro experimental que, por hipótese, tem distribuição normal e média zero.

Para a variável glicemia e insulinemia, o delineamento experimental utilizado foi o de blocos inteiramente casualizados, com parcelas subdivididas no tempo, sendo o modelo estatístico:

$Y_{i j k}=\mu+a_{i}+r_{j}+e_{i j}+b_{k}+a b_{i j k}+e_{i j k}$, em que:

$\mathrm{Y}_{\mathrm{ijk}}=$ observação da variável referente à subparcela $\mathrm{k}$ da parcela i no bloco $\mathrm{j}$;

$\mu=$ média geral;

$\mathrm{a}_{\mathrm{i}}=\mathrm{o}$ efeito da dieta $\mathrm{i}$ da parcela $(\mathrm{i}=1,2,3,4)$;

$r_{j}=o$ efeito do bloco $j(j=1,2,3,4,5,6)$;

$\mathrm{e}_{\mathrm{ij}}=\mathrm{o}$ erro experimental associado às parcelas que, por hipótese, tem distribuição normal e média zero; $\mathrm{b}_{\mathrm{k}}=$ efeito do nível $\mathrm{k}$ do fator $\mathrm{B}$ (tempo de colheita) da subparcela (com $\mathrm{k}=1,2,3,4,5,6,7,8,9,10,11$, $12,13,14,15,16,17$ para glicemia e $\mathrm{k}=1,2,3,4,5,6,7$ para insulinemia);

$a b_{i j k}=$ efeito da interação do fator A com o fator B; $\mathrm{e}_{\mathrm{ijk}}=\mathrm{o}$ erro experimental associado às subparcelas que, por hipótese, tem distribuição normal e média zero.

\section{RESULTADOS E DISCUSSÃO}

\section{Digestibilidade da matéria seca}

Os dados médios de digestibilidade da matéria seca das dietas são apresentados na Tabela 3.

Um dos motivos pelos quais a digestibilidade da matéria seca da dieta 3 (arroz) foi superior $(\mathrm{P}<0,05)$ às demais é a maior digestibilidade do amido (tabela 2). Esses resultados estão de acordo com a literatura, em que se verifica que dietas extrusadas para cães à base de arroz possuem melhor digestibilidade (KENDALL et al., 1982).

\section{Digestibilidade do amido das dietas}

Os dados médios de digestibilidade do amido total das dietas são apresentados na Tabela 3 .

Possivelmente, diferentes proporções dos componentes de amido (amilose e amilopectina) presentes na quirera de arroz, em relação aos outros cereais, bem como as reações ocorridas durante o processamento, podem ter contribuído para a maior digestibilidade do amido total da dieta 3 (arroz) em relação às demais (KENDALL et al., 1982; NUNES, 1998), embora esse fato não possa ser comprovado com os dados obtidos neste trabalho.

\section{Energia digestível das dietas}

Os dados médios de energia digestível das dietas são apresentados na Tabela 3.

As dietas 3 (arroz) e 1 (milho) destacam-se das demais pelo maior valor de energia digestível $(\mathrm{P}<0,05)$. No caso da dieta 1 (milho), a provável causa é o maior teor de óleo do grão de milho. Para a dieta 3 (arroz), esse resultado é o reflexo da maior digestibilidade do amido (Tabela 2).

TABELA 3 - Digestibilidade da matéria seca, digestibilidade do amido total e energia digestível de diferentes dietas para cães.

\begin{tabular}{lccc}
\hline Dietas experimentais & $\begin{array}{c}\text { Digestibilidade da } \\
\text { matéria seca (\%) }\end{array}$ & $\begin{array}{c}\text { Digestibilidade do } \\
\text { amido total (\%) }\end{array}$ & Energia digestível (Kcal/kg MS) \\
\hline Dieta 1 (Milho) & $82,94 \mathrm{~b}$ & $95,61 \mathrm{~b}$ & $3699 \mathrm{a}$ \\
Dieta 2 (Sorgo) & $81,11 \mathrm{~b}$ & $94,68 \mathrm{~b}$ & $3629 \mathrm{~b}$ \\
Dieta 3 (Arroz) & $87,43 \mathrm{a}$ & $98,12 \mathrm{a}$ & $3740 \mathrm{a}$ \\
Dieta 4 (Mistura) & $82,68 \mathrm{~b}$ & $95,10 \mathrm{~b}$ & $3655 \mathrm{~b}$ \\
\hline
\end{tabular}

Médias seguidas por letras distintas diferem entre si pelo teste de $\operatorname{Scott}-\operatorname{Knott}(\mathrm{P}<0,05)$. 


\section{Glicemia}

Os resultados da glicemia não apresentaram diferença significativa entre as dietas $(\mathrm{P}=0,51)$ ou interação tempo de colheita e dieta $(\mathrm{P}=0,17)$ pelo teste $\mathrm{F}$. Na Tabela 4 apresentam-se os valores de glicemia médios de 0 a 10,5 horas, após a alimentação, dos animais que receberam cada dieta, em $\mathrm{mg} / \mathrm{dL}$.

Segundo Eukanuba (2003), a digestão lenta dos carboidratos, tanto do sorgo quanto da cevada, resultariam em moderados níveis de glicose após as refeições. Entretanto, esses resultados experimentais não confirmam essa informação, uma vez que não há diferença significativa entre as dietas $(\mathrm{P}>0,05)$. As curvas glicêmicas dos animais que receberam as dietas são apresentadas na Figura 1.

A diferença na glicemia, em relação ao tempo de colheita, foi significativa $(\mathrm{P}<0,01)$ pelo teste $\mathrm{F}$ (Figura 1).

Pelos dados médios de glicemia encontrados em diferentes tempos, verifica-se que os maiores valores de glicemia estão nos tempos 30,45 e 60 minutos após a alimentação $(\mathrm{P}<0,05)$. Esse resultado já era esperado, correspondendo ao pico da maior quantidade de glicose absorvida na primeira hora após a ingestão (CUNNINGHAN, 1999; GUYTON, 1997).

Outros três tempos (3 horas, 1,5 hora, zero hora) apresentam elevados valores de glicemia, inferiores estatisticamente aos três primeiros, porém, superiores $(\mathrm{P}<0,05)$ aos demais tempos de colheita. A glicemia do

tempo 1,5 hora é apenas a descendência do primeiro pico de glicose que ocorre de 0,5 a 1,0 hora, sinalizando a entrada da glicose para as células pela ação da insulina, associada a uma menor quantidade de glicose absorvida. A glicemia do tempo 3,0 horas corresponde a um pico de absorção secundário de glicose, provavelmente oriunda da absorção final da quebra da amilose. Nos dois tempos, 1,5 e 3,0 horas, é verificada uma glicemia que corresponde a uma situação normal do organismo após a alimentação.

Já a glicemia verificada no tempo zero hora (jejum) foge à fisiologia normal da digestão, em que o animal em jejum deveria ter valores menores de glicose, dentro da euglicemia. Entretanto, o tempo zero representa o segundo contato com o animal. $\mathrm{O}$ primeiro ocorreu cerca de 10 a 15 minutos antes, quando foram coletadas as fezes defecadas durante a noite e é ainda a primeira contenção para venopunção feita no dia. Portanto, o fato de a glicemia em jejum ser maior estatisticamente $(\mathrm{P}<0,05)$ que outros tempos de colheita pode ser o reflexo de uma "descarga adrenérgica" devido ao estresse (BEHREND \& GRECO, 2000a). Apesar de todos os cuidados em conter o animal, entre eles o de o tratador de costume apreendê-lo e vendar seus olhos com a mão antes da equipe se aproximar e trabalhar em silêncio e com calma, pode ter havido influência do manejo dos animais nos resultados.

\section{Insulinemia}

Apesar de na Figura 2 se apresentar uma aparente diferença gráfica entre as curvas de insulinemia, não houve diferença significativa entre as dietas $(\mathrm{P}=0,66)$ pelo teste $\mathrm{F}$ (Tabela 4). Também não é significativa a interação tempo de colheita e dieta $(\mathrm{P}=0,97)$ para a variável insulinemia de 0 a 180 minutos, inferindo-se que os valores de insulinemia são semelhantes, independentemente do tempo de colheita.

Pelos dados, inferem-se que, estatisticamente, o pico de insulinemia, bem como a insulinemia observada de 0 a 180 minutos, não possui diferença significativa, independentemente da fonte de carboidrato utilizada na dieta (milho, sorgo, quirera de arroz ou uma mistura destes em partes iguais). Talvez as condições impostas pela extrusão (temperatura, pressão e atrito) possam ter causado tal proximidade entre as dietas, uma vez que esses cereais possuem diferenças na composição do amido e era esperada uma diferença entre as curvas insulinêmicas. No entanto, existe diferença significativa nos valores médios de insulinemia, de 0 a 3,0 horas, entre os tempos de colheita $(\mathrm{P}<0,0001)$, apresentados na Figura 2.

Esses resultados indicam que ocorre uma elevação na insulinemia aos 30 minutos após a alimentação, mais evidenciada aos 45 minutos. Porém, não se pode inferir sobre o tempo de aparecimento do pico ou sua duração, pois só foram realizadas análises até 3,0 horas, momento que, estatisticamente, ainda não houve o declínio nos níveis de insulina.

Existe uma relação entre a curva de insulinemia média e a curva glicêmica média (Figura 3), indicando uma coerência entre os resultados experimentais e entre os métodos de análise de insulina e glicose utilizados. Essa simetria já era esperada, pois o principal fator na liberação de insulina pelo pâncreas é a elevação da glicose no sangue.

Talvez uma análise dos picos de insulina, bem como da extensão do seu platô, possa trazer informações pertinentes. Assim, a avaliação da insulinemia, num futuro experimento, deveria ser realizada em tempos maiores que 180 minutos. Em contrapartida, não foi encontrada na literatura consultada nenhuma menção em relação a isso. 
TABELA 4 - Valores médios de glicemia $(\mathrm{em} \mathrm{mg} / \mathrm{dL})$ de 0 a 10,5 horas e de insulinemia $(\mu \mathrm{UI} / \mathrm{mL})$ de 0 a 3,0 horas, de cada dieta experimental.

\begin{tabular}{lcc}
\hline \multicolumn{1}{c}{ Dietas experimentais } & Glicemia & Insulinemia \\
\hline Dieta 1 (milho) & 52,10 & 14,05 \\
Dieta 2 (sorgo) & 52,08 & 15,95 \\
Dieta 3 (arroz) & 56,50 & 13,67 \\
Dieta 4 (mistura) & 55,20 & 15,29 \\
\hline
\end{tabular}

$(\mathrm{P}>0,05)$.

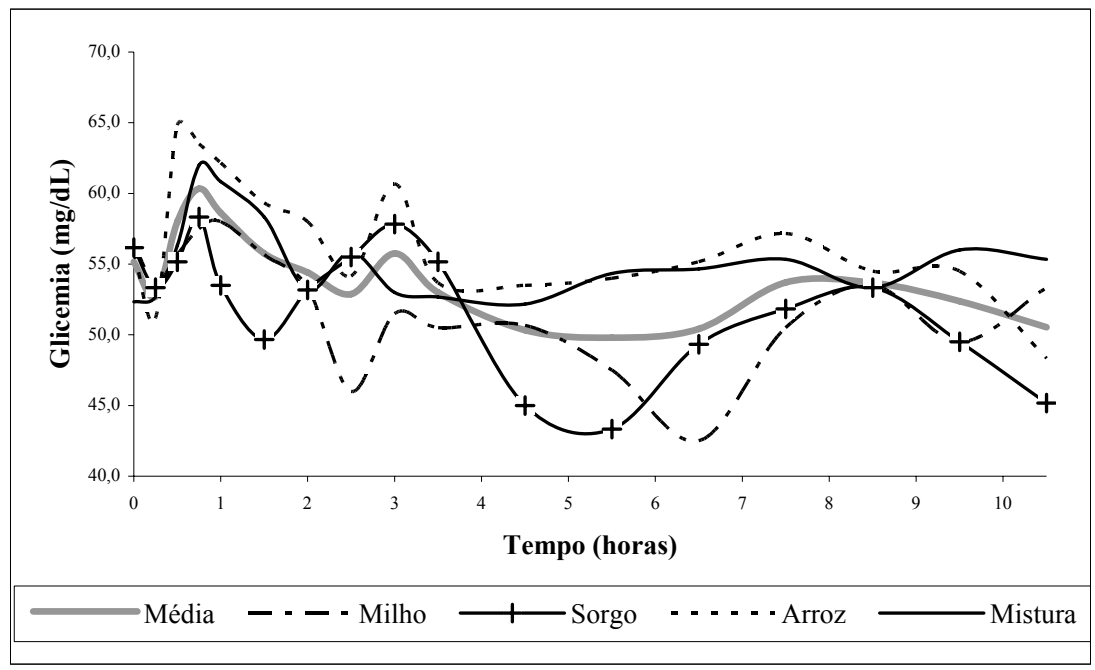

FIGURA 1 - Gráfico representando as curvas glicêmicas, em mg/dL, dos animais que receberam cada dieta, de 0 a 10,5 horas, após a alimentação.

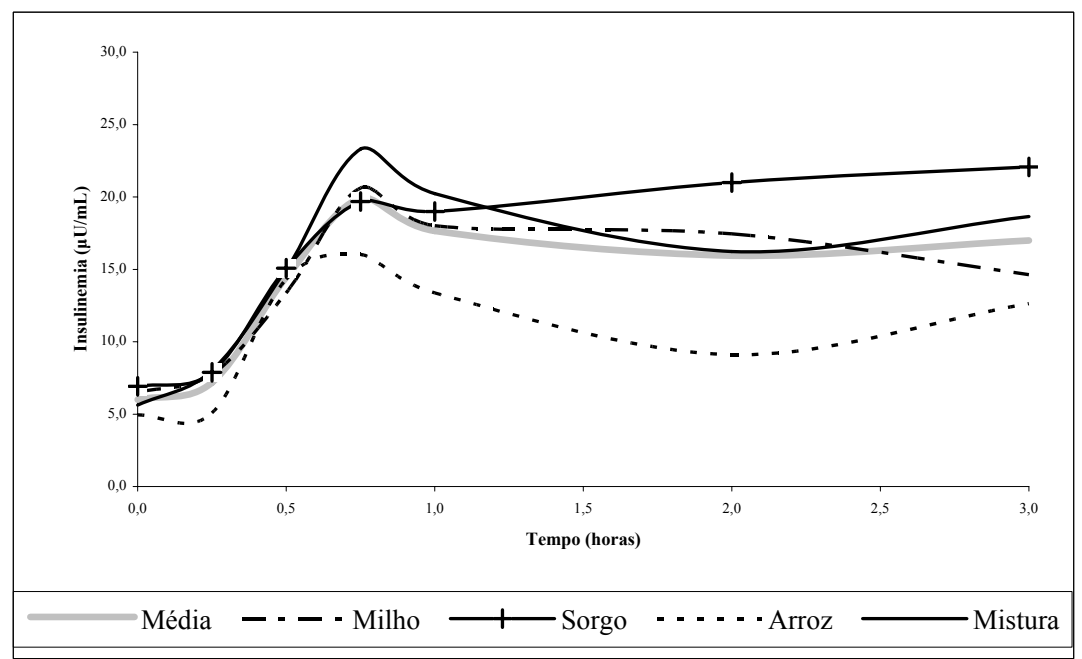

FIGURA 2 - Curvas insulinêmicas apresentando os valores médios dos animais que receberam as diferentes dietas, expressos em $\mu \mathrm{U} / \mathrm{mL}$, de 0 a 3,0 horas. 


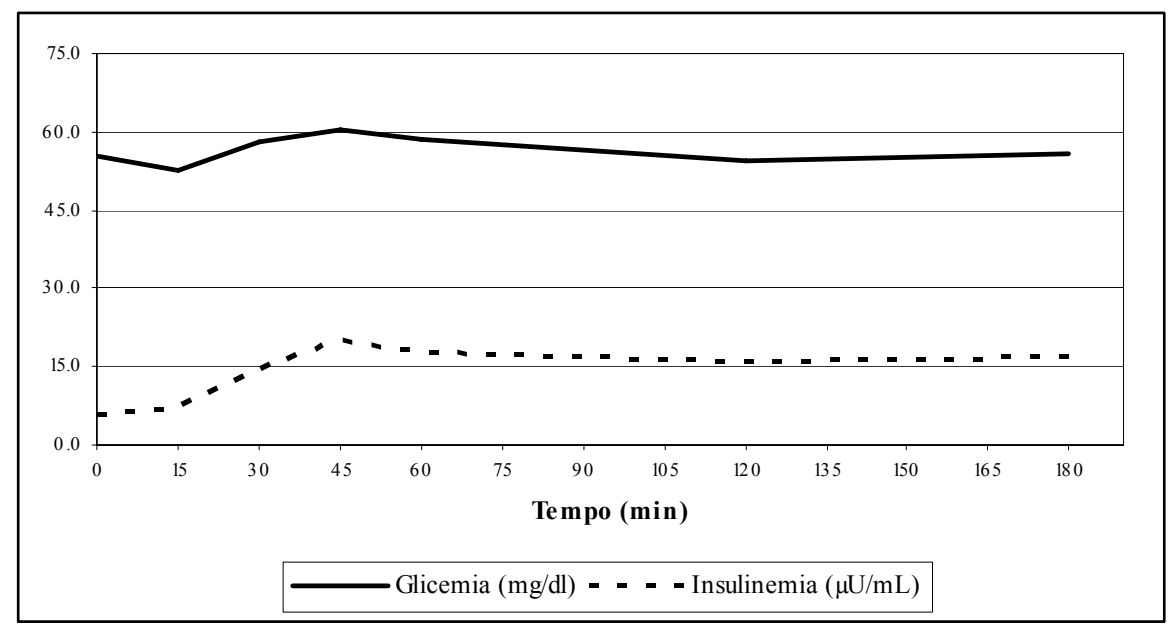

FIGURA 3 - Curvas da glicemia média $(\mathrm{mg} / \mathrm{dL})$ e insulinemia média $(\mu \mathrm{U} / \mathrm{mL})$ de 0 a 3,0 horas de animais que receberam dietas com diferentes fontes de carboidratos.

\section{CONCLUSÕES}

Apesar de a digestibilidade da matéria seca e do amido total da dieta 3 (quirera de arroz) ter sido superior às demais dietas experimentais, não há diferença significativa na glicemia ou insulinemia em relação às demais dietas experimentais.

As indústrias de alimentos extrusados para cães podem ter maior liberdade na utilização do cereal que estiver com maior disponibilidade no momento, sem alterar as características de seu produto no que tange à glicemia e insulinemia.

\section{REFERÊNCIAS BIBLIOGRÁFICAS}

ASSOCIATION OF OFFICIAL AGRICULTURAL CHEMISTIS. Official methods of analysis. 14. ed. Washington, 1984. $1141 \mathrm{p}$.

BEHREND, E. N.; GRECO, D. S. Feline diabetes mellitus: evaluation of treatment. Compendium on Continuing Education of the practicing Veterinarian, Trenton, v. 22, n. 5, p. 440-451, 2000a.

BEHREND, E. N.; GRECO, D. S. Treatment of feline diabetes mellitus: overview and therapy. Compendium on Continuing Education of the practicing Veterinarian, Trenton, v. 22, n. 5, p. 423-439, $2000 \mathrm{~b}$

COHN, L. A. et al. Assessment of five portable blood glucose meters, a point-of-care analyzer, and color test strips for measuring blood glucose concentration in dogs. Journal of the American Veterinary Medical Association, Schaumburg, v. 216, n. 15, p. 198-202, Jan. 2000.

CUNNINGHAN, J. C. Tratado de fisiologia veterinária. 2. ed. Rio de Janeiro: Guanabara Koogan, 1999. $528 \mathrm{p}$.

EUKANUBA. Eukanuba Information. 2003. Disponível em: $\quad<$ http:/www.goldenretrievers.co.nz/eukanuba_info.htm $>$. Acesso em: 15 dez. 2003.

FURTADO, D. F. Produtos tecnológicos desenvolvidos. 2003. Disponível em: $<$ http://www.dex.ufla.br/danielff/prog.htm>. Acesso em: 26 jan. 2004.

GUYTON, A. C. Tratado de fisiologia médica. 8. ed. Rio de Janeiro: Guanabara Koogan, 1997. 926 p.

HEUSNER, A. A. Energy metabolism and body size I: is the 0,75 mass exponent of Kleiber's equation a statistical artifact? Respiration Physiology, Amsterdam, v. 48, n. 1, p. 1-12, 1982.

KENDALL, P. T.; HOLME, D. W.; SMITH, P. M. Comparative evaluation of net digestive and absorptive efficiency in dog and cats fed a cariety of contrasting diets types. Journal of Small Animal Practice, London, v. 23, n. 9, p. 577-587, Sept. 1982. 
NATIONAL RESEARCH COUNCIL. Nutrient 2003, Belo Horizonte. Anais... Belo Horizonte: requirements of dogs. Washington: National Academy, $1985.79 \mathrm{p}$.

ANCLIVEPA, 2003. CD-ROM.

NOGUEIRA, R. B.; SILVA JÚNIOR, J. W.;

RAND, J. S. Management of feline diabetes: clinical CHIURCIU, J. L. V. Métodos de contenção física, colheita de efusões cavitárias e de administração de medicamentos em cães e gatos. Lavras: UFLA, 2002.

NUNES, I. J. Nutrição animal básica. 2. ed. Belo Horizonte: FEP-MVZ, 1998. 388 p. review: part 2. Australian Veterinary Practitioner, Bondi, v. 27, n. 2, p. 68-78, June 1997.

TER MAATEN, J. C. et al. Renal handling of urate and sodium during acute physiological hyperinsulinaemia in helthy subjects. Clinical Science, London, v. 92, n. 1, p. 51-58, Jan. 1997.

PEREIRA, R. L. et al. Comparação entre métodos de avaliação da glicose sérica em cães: advantage accucheck vs teste enzimático em método de ponto final. In: CONGRESSO BRASILEIRO DA ANCLIVEPA, 24.,

TORR, A. J. van et al. Experimental induction of fasting hypoglycaemia and fatty liver syndrome in three Yorkshire terrier pups. The Veterinary Quarterly, Dordrecht, v. 13, n. 1, p. 16-23, 1991. 